\title{
STUDIO DEL TRANSITORIO NELLA TRASMISSIONE DALLA P.D.P. AL VENTILATORE DI UN NEBULIZZATORE
}

\author{
Dario Friso
}

\section{INTRODUZIONE}

\section{Introduction}

Nei trattamenti ai vigneti e ai frutteti, oltre ai tradizionali atomizzatori con ventilatore assiale, possono essere impiegati, anche se meno diffusamente, i nebulizzatori dotati di ventilatore centrifugo, in grado di fornire più elevate pressioni e quindi maggiore energia cinetica all'aria. In taluni casi questa ultima è usata anche per frantumare il liquido di trattamento, in altri solo per convogliare le goccioline comunque formate da ugelli in pressione.

Ebbene durante l'impiego di una determinata serie di questi nebulizzatori si sono verificati danni al moltiplicatore, dove questo ultimo è necessario per aumentare il regime dai valori della p.d.p. a quelli richiesti dal ventilatore, dell'ordine di $420 \mathrm{rad} / \mathrm{s}$ (4000 $\left.\min ^{-1}\right)$.

I danni hanno riguardato la rottura di alcuni denti degli ingranaggi, consentendo di ipotizzare la trasmissione di una coppia eccessiva rispetto a quella di progetto del moltiplicatore stesso.

Nella presente memoria si è voluto approcciare il problema da un punto di vista teorico approntando un'analisi dinamica del transitorio che si verifica, con la frizione della p.d.p. innestata, dal regime minimo a quello nominale per la p.d.p. a 56,5 rad/s $\left(540 \mathrm{~min}^{-1}\right)$, poiché è in questa fase di regime variabile che si possono verificare sovrasforzi rispetto ai valori richiesti dal ventilatore a rotazione nominale ed è quella nella quale praticamente si sono verificate le rotture. Con i risultati dell'analisi teorica si è potuto realizzare una sperimentazione numerica, quindi quantificare l'andamento della coppia effettivamente trasmessa durante il transitorio e, infine, indicare tra le possibili soluzioni, quella ottimale consistente nell'adottare un limitatore di coppia [1, 2, 3, 4 e 5].

Memoria presentata il 05.04.2006; accettata il 22.11.2006

Ing. DARIO FRISO, professore associato confermato, dario.friso@ unipd.it., Dipartimento Territorio e Sistemi Agro-forestali, Università degli Studi Padova.

\section{MATERIALI E METODI}

\section{Materials and methods}

Il nebulizzatore in questione (fig. 1) era caratterizzato da un ventilatore centrifugo a pale rivolte all'indietro in grado di elaborare una portata di 13.000 $\mathrm{m}^{3} / \mathrm{h}$ ed una pressione totale di $7.000 \mathrm{~Pa}$ ad un regime corrispondente di $424 \mathrm{rad} / \mathrm{s}\left(4.050 \mathrm{~min}^{-1}\right)$. La potenza meccanica richiesta dal ventilatore $P_{v}$ era di $31,5 \mathrm{~kW}$, mentre il moltiplicatore, privo di limitatore di coppia e con una rapporto di 1:7,5, poteva trasmettere una potenza massima $P_{m}$ di $33 \mathrm{~kW}$ corrispondente ad una coppia massima in uscita di $77,8 \mathrm{Nm}$ a $424 \mathrm{rad} / \mathrm{s}$.

Il nebulizzatore, dotato anche di una pompa volumetrica che richiedeva una potenza $P_{p}$ di $3,5 \mathrm{~kW}$, era accoppiato ad una trattore da frutteto $4 \mathrm{RM}$ della potenza $P_{M}$ di $62,5 \mathrm{~kW}$ a $262 \mathrm{rad} / \mathrm{s}\left(2500 \mathrm{~min}^{-1}\right)$ e una coppia massima $C_{M}$ di $169,5 \mathrm{Nm}$ a $178 \mathrm{rad} / \mathrm{s}(1700$ $\left.\min ^{-1}\right)$.

\subsection{ANALISI DINAMICA}

\subsection{Dynamical analysis}

Le leggi della similitudine applicate al ventilatore consentono di scrivere che la potenza di natura fluidodinamica richiesta dalla girante è proporzionale al cubo del regime e quindi la corrispondente coppia richiesta, $C_{v}$, risulta proporzionale al quadrato dello stesso:

$$
C_{v}=k_{v} \cdot \omega_{v}^{2}
$$

dove: $\omega_{v}$ è la velocità angolare del ventilatore $(\mathrm{rad} / \mathrm{s})$; $k_{v}$ è la costante di proporzionalità determinabile introducendo nella precedente il valore di $\omega_{v n}$ pari a 424 $\mathrm{rad} / \mathrm{s}$ e di $C_{v}=P_{v} / \omega_{v n}=31,5 \cdot 10^{3} / 424=74,2^{v n} \mathrm{Nm}$. Di qui il valore di $k_{v}=4,13 \cdot 10^{-4} \mathrm{Nms}^{-2}$.

Se si suppone, come in sostanza avviene nella pratica, che il trattore sia fermo durante la fase di transitorio cioè di accelerazione della p.d.p. dal minimo alla velocità nominale, la coppia motrice $C_{m}$ disponibile alla girante, è quella del motore $C_{M}$, ridotta dal rendimento totale di trasmissione $\eta_{t}$, posto pari a 0,92 , me- 
no quella richiesta dalla pompa volumetrica del liquido di trattamento $C_{p}$. Poiché anche una tale pompa chiede una coppia proporzionale al quadrato del proprio regime e quindi anche al quadrato del regime del ventilatore, dato che tra i due esiste un rapporto di trasmissione fisso, per essa vale la:

$$
C_{p}=k_{p} \cdot \omega_{v}^{2}
$$

dove: $k_{p}$ è la costante di proporzionalità determinabile introducendo nella precedente il valore di $\omega_{v n}$ pari a $424 \mathrm{rad} / \mathrm{s}$ e di $C_{p}=P_{p} / \omega_{v n_{5}}=3,5 \cdot 10^{3} / 424=8,2 \mathrm{Nm}$. Di qui il valore di $k_{p}=4,56 \cdot 10^{-5} \mathrm{Nms}^{-2}$.

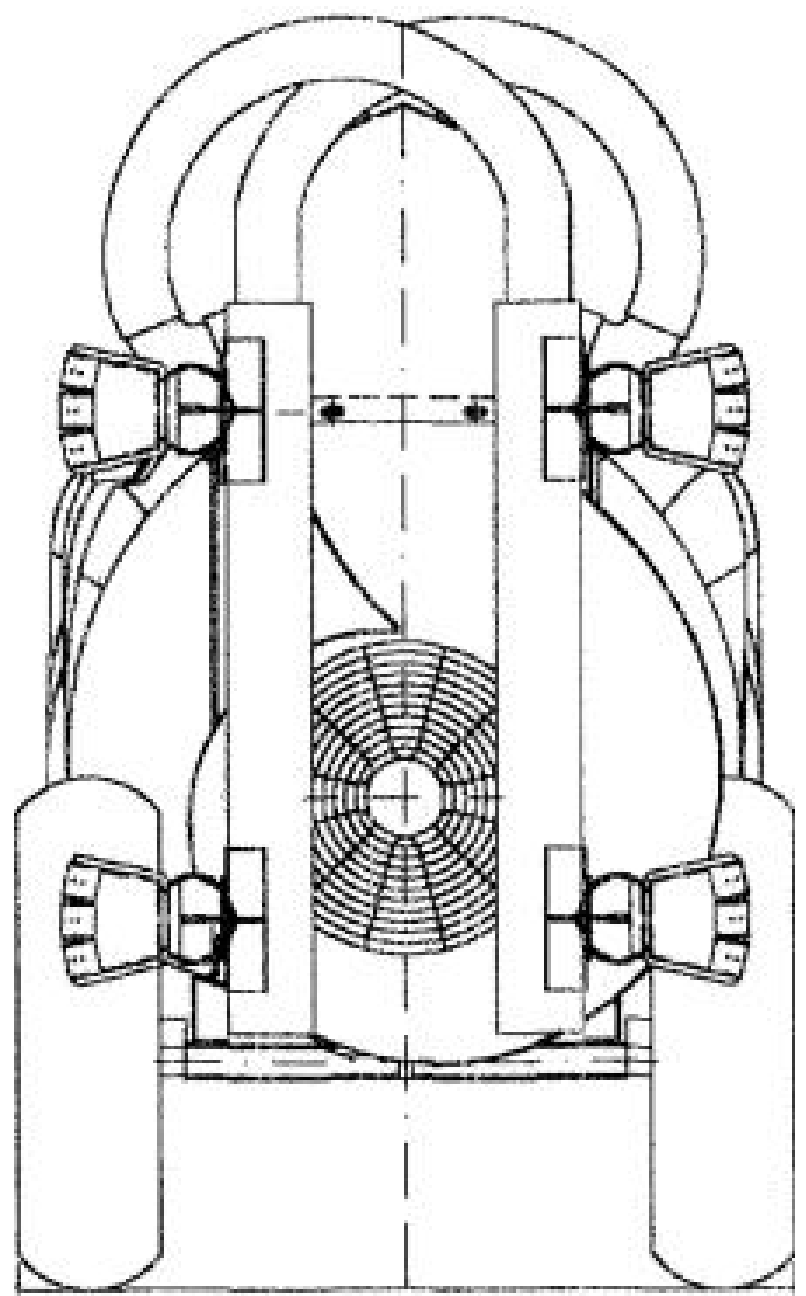

Fig. 1 - Il nebulizzatore.

Fig. 1 - The mistblower.

La coppia del motore $C_{M}$ ha il ben noto andamento rispetto al regime del motore e quindi anche rispetto al regime del ventilatore. Il legame può convenientemente essere rappresentato da una equazione di $2^{\circ}$ grado:

$$
C_{M}=c \cdot \omega_{v}^{2}+d \cdot \omega_{v}+e
$$

dove: $c, d$ ed $e$ sono costanti da determinare con una regressione parabolica.
Se la potenza del motore del trattore $P_{M}$ è di 62,5 $\mathrm{kW}(85 \mathrm{CV})$ in corrispondenza del regime di 56,5 $\mathrm{rad} / \mathrm{s}\left(540 \mathrm{~min}^{-1}\right)$ della p.d.p., la coppia del motore corrispondente al regime del ventilatore di $424 \mathrm{rad} / \mathrm{s}$, sarà $C_{M}=P_{M} / \omega_{v n}=62,5 \cdot 10^{3} / 424=147,4 \mathrm{Nm}$. Supponendo che lo stesso valore di coppia sia ragionevolmente fornito dal motore anche al regime minimo del ventilatore di $150 \mathrm{rad} / \mathrm{s}$ e considerando una riserva di coppia del $15 \%$ e una riserva di giri (motore) di 800 , si hanno le tre doppiette di valori di coppia-regime da utilizzare per la regressione della (3): (147,4-424); (169,5-273); (147,4-150). Regressione che consente di determinare le seguenti costanti: $c=-1,19 \cdot 10^{-3}$; $d=0,683 ; e=71,7$.

Si può ora impostare l'equazione della dinamica, applicata alla girante durante il transitorio, che vede questa ultima accelerare dal regime minimo a quello nominale:

$$
\begin{aligned}
& 1 \frac{d \omega}{d t}=C_{2}-C_{r}-\eta C_{\mathrm{N}}=C_{\mathrm{r}}-C_{\mathrm{r}}- \\
& =\left(n_{i} c-k_{r}-k_{r}\right)-e_{c}^{2}+n_{h} d \cdot \omega_{p}+\eta_{i}
\end{aligned}
$$

dove: I è il momento d'inerzia della girante calcolato, partendo dalla geometria e dalla massa della stessa, pari a $0,72 \mathrm{kgm}^{2}$.

La (4) è un'equazione differenziale del $1^{\circ}$ ordine non lineare la cui soluzione è:

$$
\theta_{r}=\frac{-\eta_{r} d+f \cdot T_{g h}\left[\frac{1}{2} f\left(-\frac{t}{l}+C_{\omega+1}\right)\right]}{2\left(\eta c-k_{r}-k_{r}\right)}
$$

dove: $t$ è il tempo (s); $f$ è la seguente radice:

$$
f=\sqrt{\eta_{i}^{2} d^{2}-4 \eta_{i}^{2} c \cdot c+4 \eta_{i} c\left(k+k_{y}\right)}
$$

La costante di integrazione $C_{o s t}$ è determinabile con la condizione iniziale $\omega_{v}=\omega_{v \min }=150 \mathrm{rad} / \mathrm{s}$ per $t=0$ :

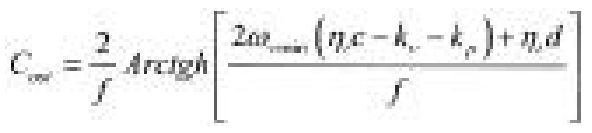

Derivando la (5) e moltiplicando per il momento d'inerzia $I$, si ottiene la coppia d'inerzia in funzione del tempo $t$ :

$$
r \frac{d \omega}{d t}=\frac{-f^{2}}{4\left(n c-k_{v}-k_{n}\right) \cdot \cos h^{2}\left[\frac{1}{2} f\left(-\frac{t}{1}+C_{-j}\right)\right]}
$$

Combinando poi la coppia fluidodinamica richiesta dal ventilatore $C_{v}$, data dalla (1), con la (5), si ottiene una nuova equazione di $C_{v}$ funzione del tempo $t$ :

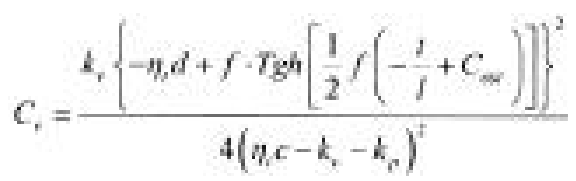

Sommando infine la (8) con la (9) si ricava l'andamento rispetto al tempo della coppia totale richiesta dal ventilatore al moltiplicatore $C_{m}$ durante il transitorio. 


\section{RISULTATI E DISCUSSIONE}

\section{Results and discussion}

Nella condizione di assenza di limitatore di coppia, l'equazione (5) unitamente alla (7), che dà la costante di integrazione a partire dalle condizioni iniziali e con i valori precedentemente trovati per le costanti $c$, $d, e, k_{p}, k_{v}$ e $\eta_{t}$, fornisce l'andamento della velocità angolare della girante $\omega_{v}$ in funzione del tempo ed è visibile in figura 2.

L'aumento graduale del regime del ventilatore si ferma al raggiungimento del valore nominale $\omega_{v n}$ previsto di $424 \mathrm{rad} / \mathrm{s}\left(4050 \mathrm{~min}^{-1}\right)$, ad opera del regolatore del motore. Qui si è ipotizzato che questo ultimo attui un intervento istantaneo di parziale chiusura dell'alimentazione del motore, alfine di ridurre la coppia motrice e quindi imporre il valore fisso di $\omega_{v n}$. È noto invece che il regolatore ha una sua sensibilità che rende l'intervento non proprio istantaneo. Tuttavia l'ipotesi fatta non inficia i risultati del transitorio precedente l'intervento del regolatore stesso.

Dalla figura 2 va anche segnalato l'intervallo di tempo del transitorio pari a $1,8 \mathrm{~s}$. Sono cioè sufficienti meno di $2 \mathrm{~s}$ per portarsi al regime nominale partendo dalla condizione di p.d.p. inserita ma a regime minimo.

In figura 3 sono stati diagrammati gli andamenti, durante il transitorio, della coppia d'inerzia $I \cdot d \omega / d t$ fornita dall'equazione (8), della coppia richiesta dalla fluidodinamica del ventilatore $C_{v}$ data dall'equazione (9) e della loro somma che è la coppia totale uscente dal moltiplicatore $C_{m}$, tutte in funzione del tempo $t$.

Si noti come nel transitorio la coppia richiesta per vincere l'inerzia sia prevalente su quella di natura fluidodinamica del ventilatore; solo verso la fine i valori diventano confrontabili. Circa la somma delle due, ovvero la coppia totale uscente dal moltiplicatore $C_{m}$, essa presenta un massimo di $159,1 \mathrm{Nm}$ e risulta influenzata in modo trascurabile dal momento d'inerzia del moltiplicatore poiché questo ultimo è 15 volte inferiore a quello della girante.

La figura 4 riporta l'andamento, sempre durante il transitorio, della potenza d'inerzia, di quella di natura fluidodinamica del ventilatore e di quella totale somma delle due, ottenute tutte moltiplicando per la velocità angolare $\omega_{v}$ del ventilatore le rispettive coppie. La curva della potenza totale trasmessa alla girante presenta un massimo alla fine del transitorio, con un valore di $54 \mathrm{~kW}$ corrispondente alla potenza nominale del motore, pari a $62,5 \mathrm{~kW}$, al netto del rendimento totale di trasmissione, pari a 0,92 , e decurtata della potenza richiesta dalla pompa di $3,5 \mathrm{~kW}$. Giova ricordare l'ipotesi avanzata nel par. 2.1 ovvero che il trattore si ritenga fermo durante l'accelerazione del ventilatore e, inoltre, che la potenza di targa trasmissibile del moltiplicatore è di $33 \mathrm{~kW}$.

La prima soluzione che emerge immediata per evitare danni al moltiplicatore è quella di dimensionarlo per la potenza massima ora determinata o, meglio, per

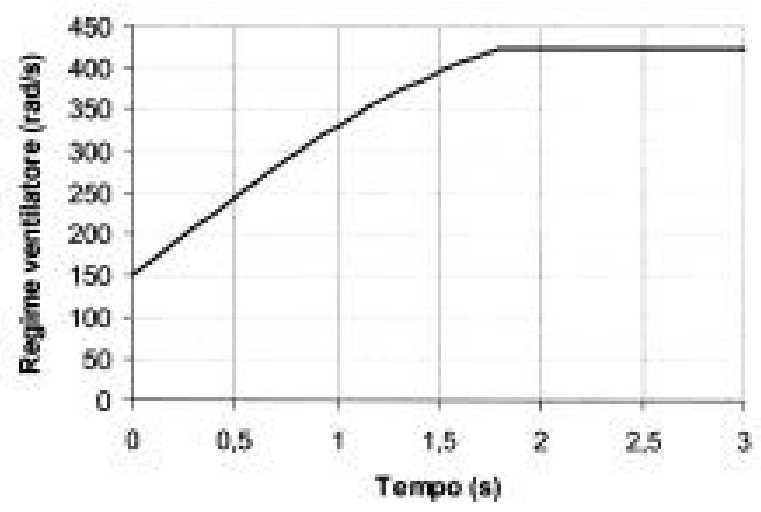

Fig. 2 - Regime del ventilatore $\omega_{\mathrm{v}}$ in funzione del tempo $\mathrm{t} d u$ rante il transitorio con girante in acciaio e senza limitatore di coppia.

Fig. 2 - Fan speed $\omega_{v}$ vs. time $t$ during the no steady-state condition with steel impeller and without torque limiter.

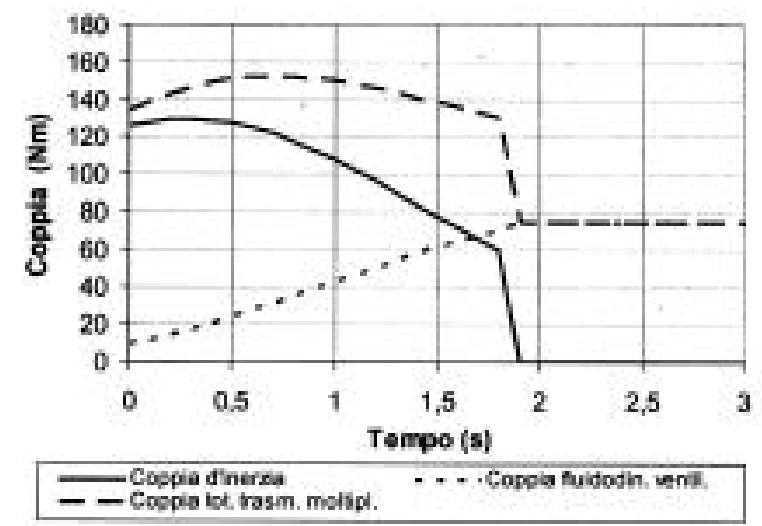

Fig. 3 - Coppia d'inerzia, coppia fluidodinamica del ventilatore e coppia totale trasmessa dal moltiplicatore in funzione del tempo durante il transitorio con girante in acciaio e senza limitatore di coppia.

Fig. 3 - Inertial torque, fan torque and total torque gearing by overgear-box vs. time during the no steady-state condition with steel impeller and without torque limiter.

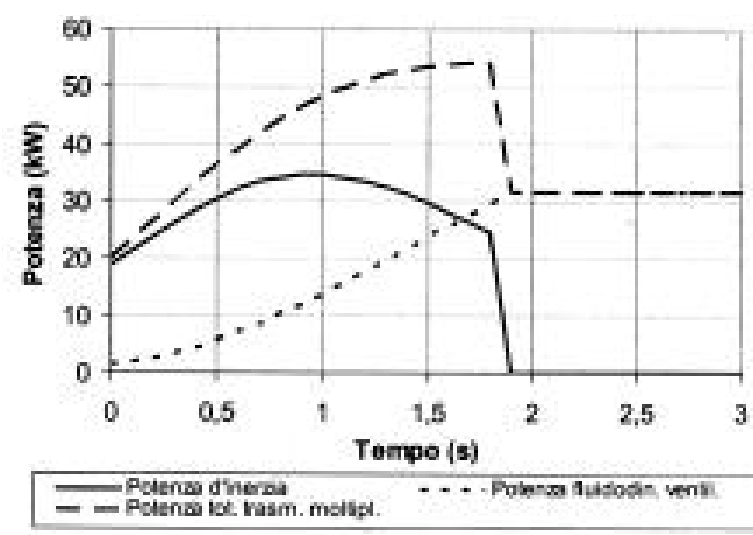

Fig. 4 - Potenza d'inerzia, potenza fluidodinamica del ventilatore e potenza totale trasmessa dal moltiplicatore in funzione del tempo durante il transitorio con girante in acciaio e senza limitatore di coppia.

Fig. 4 - Inertial power, fan power and total power gearing by overgear-box vs. time during the no steady-state condition with steel impeller and without torque limiter. 
quella nominale del motore. Direttiva di calcolo sicura ma costosa.

Una seconda soluzione è quella di installare a monte del moltiplicatore un limitatore di coppia come spesso si fa con le operatrici per la lavorazione del terreno. Per verificarla si è reimpostata l'equazione della dinamica della girante:

$$
1 \frac{d \omega}{d t}=\eta_{\omega} \cdot C_{r}-C_{r}=\eta_{a} \cdot C_{r}-k_{r}-\omega_{r}^{2}
$$

dove: $C_{l}$ è la coppia massima filtrata dal limitatore, considerata costante rispetto al regime e pari a $C_{l}=P_{m} / \omega_{v n}=33 \cdot 10^{3} / 424=77,8 \mathrm{Nm} ; P_{m}$ è la potenza di targa sopportabile dal moltiplicatore, pari a $33 \mathrm{~kW}$; $\omega_{v n}$ è la velocità angolare nominale del ventilatore pari a $424 \mathrm{rad} / \mathrm{s} ; \eta_{m}$ è il rendimento di trasmissione del moltiplicatore posto pari a 0,98 .

La soluzione dell'equazione differenziale (10), sempre del $1^{\circ}$ ordine e non lineare, risulta:

$$
a_{v}=\sqrt{\frac{\eta_{*} \cdot C_{i}}{k_{r}}}, \tau_{g} h_{[}\left[\sqrt{\eta_{m} \cdot C_{j} \cdot k_{v}}\left(\frac{t}{I}-C_{m+1}\right)\right]
$$

La costante di integrazione $C_{\text {ost }}$ si ricava con la condizione iniziale $\omega_{v}=\omega_{v \min }=150 \mathrm{rad} / \mathrm{s}$ per $t=0$ :

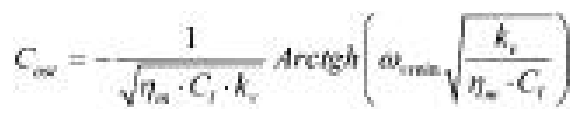

Derivando la (11) e moltiplicando per il momento d'inerzia $I$, si ottiene la coppia d'inerzia in funzione del tempo $t$ :

$$
f \frac{d \omega}{d t}=\frac{\eta_{-} \cdot C_{j}}{\cosh ^{2}\left[\sqrt{\eta_{m}-C_{r}-k_{r}}\left(\frac{t}{t}-C_{-\alpha}\right)\right]}
$$

Combinando poi la (1) con la (11), si ottiene la coppia fluidodinamica $C_{v}$ in funzione del tempo $t$ :

$$
C_{v}=\eta_{\mu} \cdot C_{i} \cdot \operatorname{Tgh}^{2}\left[\sqrt{\eta_{-} \cdot C_{i} \cdot k_{+}}\left(\frac{t}{l}-C_{\omega}\right)\right]
$$

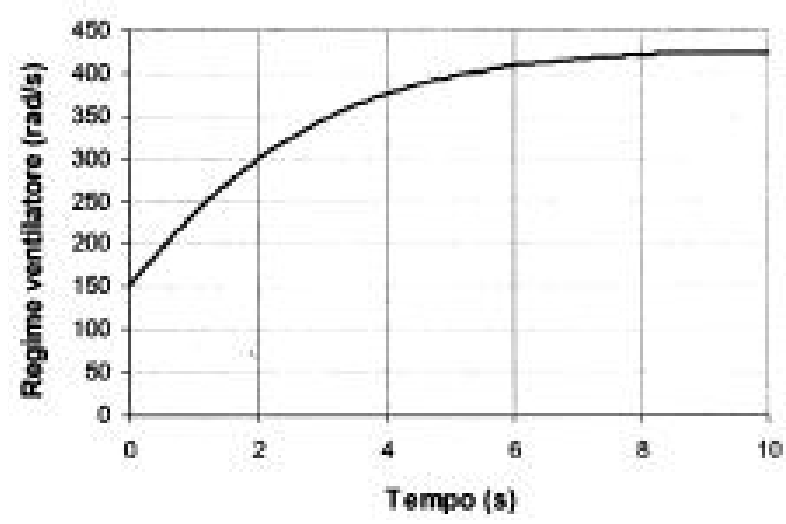

Fig. 5 - Regime del ventilatore $\omega_{v}$ in funzione del tempo $\mathrm{t} d u$ rante il transitorio con girante in acciaio e con limitatore di coppia.

Fig. 5 - Fan speed $\omega_{v}$ vs. time $t$ during the no steady-state condition with steel impeller and with torque limiter.
Sommando infine la (13) con la (14) si ricava l'andamento rispetto al tempo della coppia totale richiesta dal ventilatore e che risulta pari al valore costante della coppia filtrata dal limitatore al netto del rendimento del moltiplicatore stesso $\eta_{m} C_{l}$, come è facilmente verificabile considerando le proprietà delle funzioni iperboliche e come, d'altra parte, doveva essere in base anche alla (10).

Le figure 5 e 6 riportano rispettivamente gli andamenti del regime della girante, della coppia d'inerzia richiesta dal ventilatore, di quella fluidodinamica dello stesso e di quella totale trasmessa dal moltiplicatore, mentre la figura 7 riporta l'andamento della potenza, sempre nei due contributi d'inerzia e fluidodinamica del ventilatore, nonché quella totale del moltiplicatore.

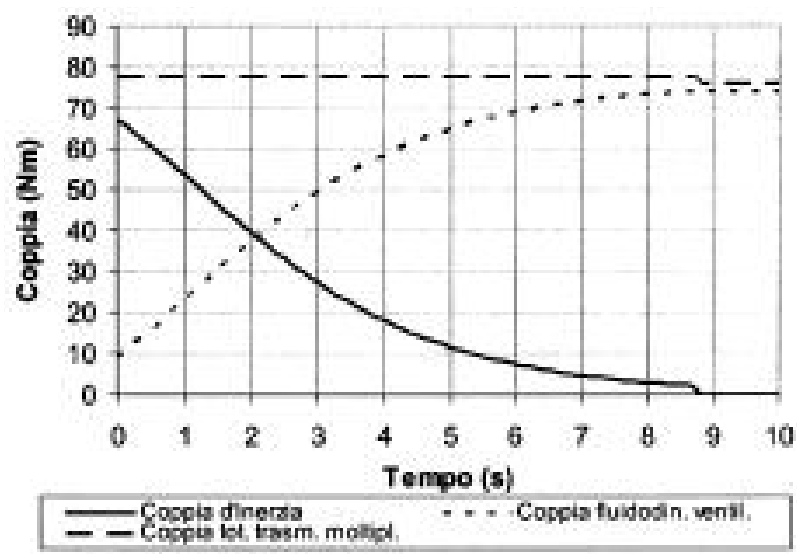

Fig. 6 - Coppia d'inerzia, coppia fluidodinamica del ventilatore e coppia totale trasmessa dal moltiplicatore in funzione del tempo durante il transitorio con girante in acciaio e con limitatore di coppia.

Fig. 6 - Inertial torque, fan torque and total torque gearing by overgear-box vs. time during the no steady-state condition with steel impeller and with torque limiter.

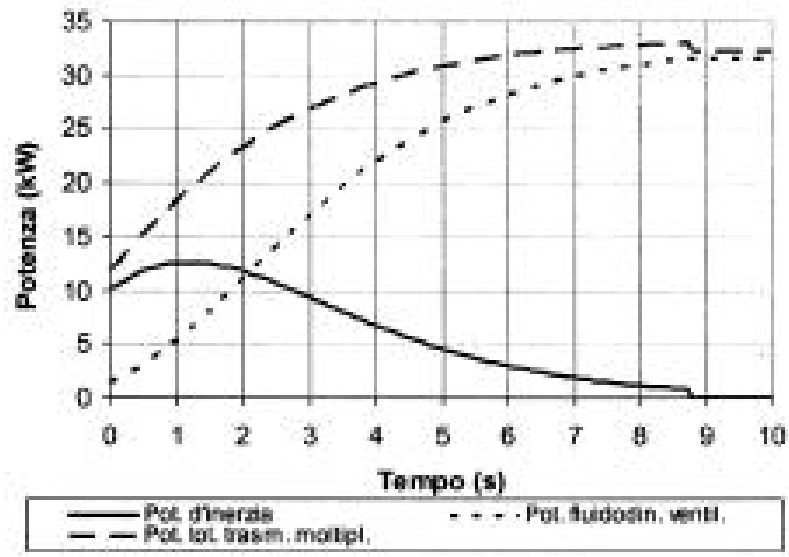

Fig. 7 - Potenza d'inerzia, potenza fluidodinamica del ventilatore e potenza totale trasmessa dal moltiplicatore in funzione del tempo durante il transitorio con girante in acciaio e con limitatore di coppia.

Fig. 7 - Inertial power, fan power and total power gearing by overgear-box vs. time during the no steady-state condition with steel impeller and with torque limiter. 


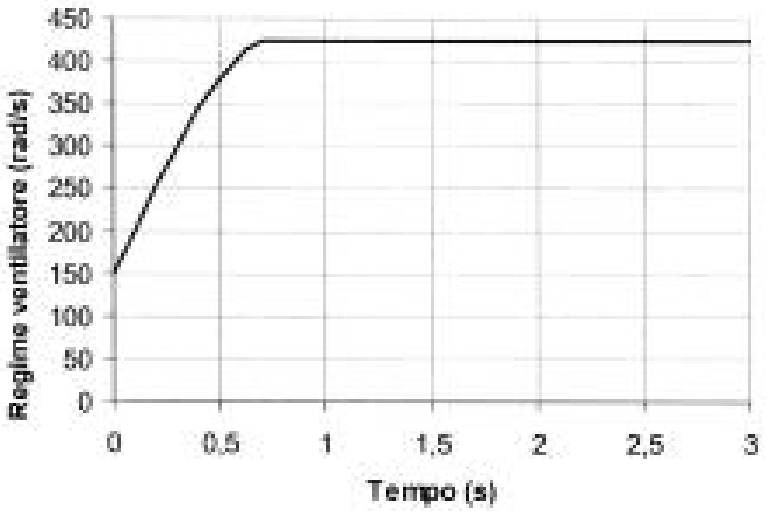

Fig. 8 - Regime del ventilatore $\omega_{v}$ in funzione del tempo $\mathrm{t} d u$ rante il transitorio con girante in alluminio e senza limitatore di coppia.

Fig. 8 - Fan speed $\omega_{v}$ vs. time $t$ during the no steady-state condition with aluminium impeller and without torque limiter.

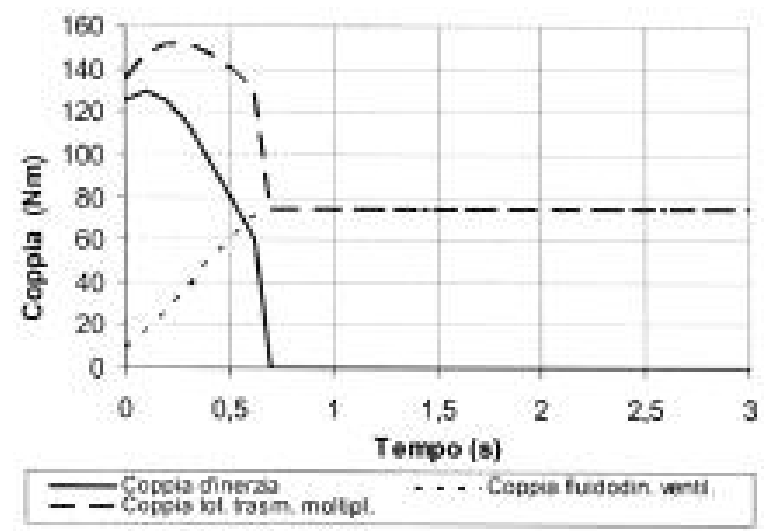

Fig. 9 - Coppia d'inerzia, coppia fluidodinamica del ventilatore e coppia totale trasmessa dal moltiplicatore in funzione del tempo durante il transitorio con girante in alluminio e senza limitatore di coppia.

Fig. 9 - Inertial torque, fan torque and total torque gearing by overgear-box vs. time during the no steady-state condition with aluminium impeller and without torque limiter.

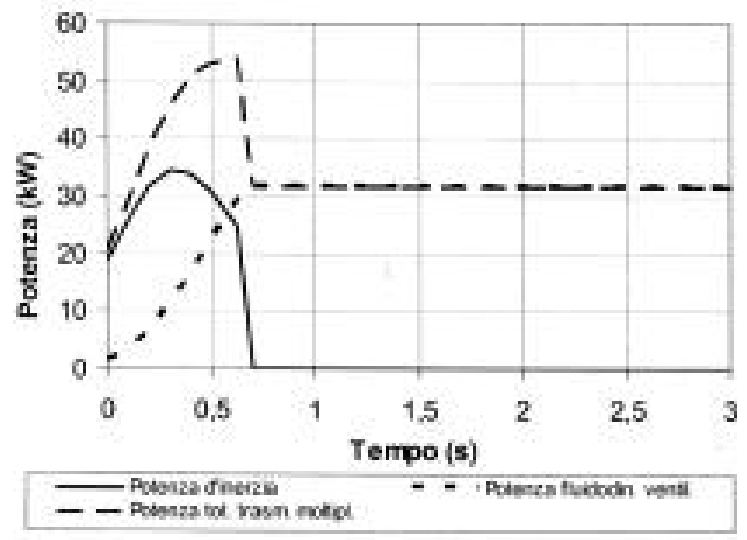

Fig. 10 - Potenza d'inerzia, potenza fluidodinamica del ventilatore e potenza totale trasmessa dal moltiplicatore in funzione del tempo durante il transitorio con girante in alluminio e senza limitatore di coppia.

Fig. 10 - Inertial power, fan power and total power gearing by overgear-box vs. time during the no steady-state condition with aluminium impeller and without torque limiter.
Come appare, viene rispettata la condizione limite per la potenza massima trasmessa di $33 \mathrm{~kW}$, valore che si raggiunge solo verso la fine del transitorio. Tuttavia va segnalato, per converso, l'allungamento del transitorio stesso che ora dura $8,7 \mathrm{~s}$, valore ancora accettabile anche se quasi 5 volte più di quello senza limitatore di coppia.

$\mathrm{Si}$ è voluto testare, sempre con una sperimentazione numerica, una terza ipotesi, ovvero quella di una riduzione del momento d'inerzia della girante tramite una costruzione in alluminio anziché in acciaio. Tenuto conto che quest'ultimo ha una massa volumica 2,88 volte superiore ( 7800 contro $2700 \mathrm{~kg} / \mathrm{m}^{3}$ ) e una resistenza meccanica circa doppia rispetto all'alluminio, una girante costruita con questo ultimo a pari geometria risulta avere dunque un momento d'inerzia $I$ parimenti inferiore $\left(0,25\right.$ contro $\left.0,72 \mathrm{kgm}^{2}\right)$. Applicando le equazioni (5), (7), (8) e (9), con questo nuovo valore di $I$, si sono ricavati gli andamenti della velocità angolare $\omega_{v}$, della coppia d'inerzia $I \cdot d \omega / d t$, di quella fluidodinamica del ventilatore $C_{v}$ e di quella totale richiesta al moltiplicatore $C_{m}$, rispetto al tempo $t$.

I risultati sono evidenziati rispettivamente nelle $\mathrm{fi}$ gure 8 e 9 , mentre nella figura 10 è rappresentato l'andamento della potenza, d'inerzia, fluidodinamica e totale richiesta al moltiplicatore. Emerge subito che questa ultima è rimasta invariata ovvero pari a quella disponibile dal motore al netto del rendimento totale di trasmissione (p.d.p. + moltiplicatore), meno quella richiesta dalla pompa, cioè $54 \mathrm{~kW}$, così come la coppia massima è quella disponibile netta dal motore, mentre a variare risulta il tempo che si riduce a $0,62 \mathrm{~s}$, esattamente di 2,88 volte inferiore rispetto a quello della girante in acciaio. Infatti esplicitando il tempo dalla (5) si evidenzia la proporzionalità diretta dello stesso con il momento d'inerzia. Dunque la riduzione del momento d'inerzia non produce l'effetto desiderato, ovvero la riduzione della coppia e quindi della potenza d'inerzia, ma solo una compattazione del tempo del transitorio.

\section{CONCLUSIONI}

\section{Conclusions}

A fronte del problema connesso alla rottura del moltiplicatore interposto tra la p.d.p. e il ventilatore centrifugo di una serie di nebulizzatori per i trattamenti a frutteti e a vigneti, si è indagato, da un punto di vista teorico, sul transitorio che si verifica, con la frizione della p.d.p. innestata, dal regime minimo a quello nominale della p.d.p. a $56,5 \mathrm{rad} / \mathrm{s}$ (540 giri/min).

L'analisi ha visto impostare l'equazione differenziale della dinamica del moto rotatorio della girante e condurre l'integrazione della stessa fino a ottenere l'andamento nel tempo della coppia e della potenza totali trasmesse dal moltiplicatore unitamente alle componenti d'inerzia e fluidodinamiche del ventilatore. 
Emerge che la superiore disponibilità di potenza da parte del motore rispetto a quella richiesta dalle condizioni fluidodinamiche del ventilatore viene tutta utilizzata per vincere l'inerzia della girante. Di qui l'inutilità dell'intervento di alleggerimento di questa ultima: comunque la potenza che attraversa il moltiplicatore rimane quella disponibile dal motore.

Anche l'ipotesi di soluzione al problema tramite il surdimensionamento del moltiplicatore sulla potenza del motore risulta poco praticabile, soprattutto per il costo, dato che bisogna far riferimento al trattore più potente disponibile nella gamma di quelli per frutteto/vigneto.

La terza soluzione ipotizzata, ovvero quella di installare un limitatore di coppia a monte del moltiplicatore, è quella invece risultata praticabile. Infatti l'analisi dinamica sul transitorio conferma il non superamento della potenza di targa del moltiplicatore, tuttavia impone un allungamento del transitorio, peraltro accettabile, quale tempo di accelerazione della girante fino al valore nominale, di quasi 5 volte $(8,7 \mathrm{~s}$ contro 1,8$)$.

\section{ELENCO DEI SIMBOLI}

\section{Nomenclature}

$c, d, e$ costanti di regressione della coppia-motore

$C_{l} \quad$ coppia massima trasmessa dal limitatore $(\mathrm{Nm})$

$C_{m} \quad$ coppia disponibile al ventilatore (Nm)

$C_{M}^{m} \quad$ coppia del motore del trattore (Nm)

$C_{p} \quad$ coppia richiesta dalla pompa (Nm)

$C_{v} \quad$ coppia richiesta dal ventilatore $(\mathrm{Nm})$

$f \quad$ costante (vedi eq. (6))

I momento d'inerzia della girante $\left(\mathrm{kgm}^{2}\right)$

$k_{p} \quad$ costante di proporzionalità della pompa $\left(\mathrm{Nms}^{-2}\right)$

$k_{v} \quad$ costante di proporzionalità del ventilat. $\left(\mathrm{Nms}^{-2}\right)$

$P_{m} \quad$ potenza trasmissibile dal moltiplicatore $(\mathrm{kW})$

$P_{M}^{m} \quad$ potenza del motore $(\mathrm{kW})$

$P_{p}^{M} \quad$ potenza richiesta dalla pompa $(\mathrm{kW})$

$P_{v}^{p} \quad$ potenza richiesta dal ventilatore $(\mathrm{kW})$

$t \quad$ tempo (s)

$\eta_{m} \quad$ rendimento di trasmissione del moltiplicatore

$\eta_{t} \quad$ rendimento totale di trasmissione dal motore al ventilatore

$\omega_{\min } \quad$ velocità angolare minima del ventilatore $(\mathrm{rad} / \mathrm{s})$

$\omega_{v} \quad$ velocità angolare variabile ventilatore $(\mathrm{rad} / \mathrm{s})$

$\omega_{v n} \quad$ velocità angolare nominale ventilatore $(\mathrm{rad} / \mathrm{s})$

\section{BIBLIOGRAFIA}

\section{References}

[1]Chestney A.W., Crolla D.A., Overload clutch materials for agricultural p.t.o. driven machinery, J. Agri. Eng. Res. (1979) 24 (2), 183-198.

[2] Chestney A.W., The development and evaluation of a combined overload and overrun clutch for agricultural p.t.o. driven machinery, J. Agri. Eng. Res. (1984) 29 (1), 35-42.

[3]Crolla D.A., Theoretical analysis of inertia torque overloads when starting up p.t.o. driven agricultural machinery, J. Agri. Eng. Res. (1977) 22 (2), 197-208.

[4]Crolla D.A., Chestney A.W., The performance of friction materials in overloads clutches, J. Agri. Eng. Res. (1978) 47 (1), 165-184.

[5]Crolla D.A., Chestney A.W., Field measurements of driveline torque imposed on p.t.o. driven machinery, J. Agri. Eng. Res. (1979) 24 (2), 157-181.

Parole chiave: analisi dinamica, transitorio, ventilatore, moltiplicatore, nebulizzatore.

\section{SUMMARY}

\section{STUDY OF NO STEADY-STATE CONDITION ON GEARING FROM THE P.T.O. TO THE FAN OF MISTBLOWERS}

During the use of a number of mistblowers for the orchard and vineyard protection, the overgear-box, located between the p.t.o. and the centrifugal fan, was damaged.

To solve the problem we carried-out a dynamic analysis of initial no steady-state by integrating the differential equation of the impeller motion. By the obtained equations, a numerical experimentation was carried-out. It showed the gearing of a power greater than design power of the overgear box, due to impeller inertia. This result explains the overgear box damages and gave the no steady-state condition time $(1,8 \mathrm{~s})$, to get the rated velocity of the impeller.

Finally a torque limiter assembling, between the p.t.o. and the overgear box, was proposed. A new differential equation solution and a new numerical experimentation was carried-out. It showed a reduction of the geared power to the rated value of the overgearbox, together with an increase of the no steady-state condition time to $8,7 \mathrm{~s}$.

Key words: dynamic analysis, no steady state condition, fan, overgear box, mistblower. 